\title{
Customers' Emotions in Service Failure and Recovery: A Meta-Analysis
}

\begin{abstract}
.
Customers' emotions have emerged as one of the dominant dimensions in the complaint-handling domain. This research provides a quantitative synthesis of the role of emotions triggered by service failure/recovery situations. First, we outline a conceptual framework showing when emotions play a role in service-recovery situations and the key constructs affected by emotions. Second, we show that existing studies represent emotions using either dimensional or discrete models, and that this distinction significantly affects the strength of the relationships involving emotions. Third, we show that the perceived fairness of procedures is more powerful than distributive and interactional justice in triggering both negative and positive emotions. Fourth, our moderator analysis shows that methodological and measurement characteristics, along with culture, explain systematic patterns of variation in the effect sizes. Finally, our managerial moderator analysis shows how different forms of servicerecovery actions, such as cash compensation, product or service replacement, and time needed to manage the recovery, affect the strength of the relationships involving emotions
\end{abstract}

Keywords: Meta-analysis, Complaint handling, Service failure emotions

Conference Track: Services Marketing 
The last fifteen years of complaint handling research have witnessed a rapid growth in the number of empirical studies that have included customers' emotional responses following service failure and recovery. This evidence suggests that emotions are key factors related to cognitive reactions of customers to service recovery efforts (i.e. perceived justice dimensions), and to relevant outcome variables such as loyalty, customer satisfaction, return intent, word of mouth, and intention to complain. Despite the significant number of empirical work, insights from this stream of research have not always been cumulative. Overall, these findings indicate that the impact of emotions may vary depending on the theoretical and methodological choices of the studies, the way emotions are measured, and the cultural orientation. Indeed, studies conceptualize emotions following either a discrete or a dimensional model, conduct experiments or observational studies, measure emotions using different scales, and collect data in different countries and cultures.

This suggests the need for a meta-analysis to integrate the evidence of accumulated empirical research. This represents the purpose of this research. More specifically, through metaanalysis we aim to: 1) reflect on the theoretical conceptualization of emotions in service recovery domain, 2) map the constructs that have been examined in relation to emotions, 3) identify which of these constructs are more strongly related to emotions, and 4) assess the role of moderating variables in shaping the magnitude of the relationships.

\section{Conceptual review}

Cognitive appraisal theory (CAT, Lazarus 1966) is the prevailing theory to theoretically ground the mechanism through which negative or positive service episodes trigger customer emotional reactions. Emotions are defined as mental states that arise from the cognitive appraisal of the service situation (Smith and Bolton 2002). Thus, the subjective appraisal of a failure or recovery episode for a customer generates the emotional state. Complaint handling studies also distinguish between discrete and valence models of emotions (Barret 1998). Discrete models assume that emotions are best represented as discrete constructs that differ one from the other and tend to have unique effects on cognition and judgment. Accordingly, emotions such as sadness and anger are expected to exert different effects on outcomes. By contrast, valence models (i.e., positive vs. negative emotions) assume that emotions of the same valence have similar effects on outcomes. Irrespective of the emotion model adopted by scholars, complaint handling research agrees on the correlates of positive and negative emotions. Positive (negative) emotions are positively (negatively) related to distributive, interactional, and procedural justice, transactional and overall satisfaction, behavioral and attitudinal loyalty, positive WOM intent and trust. In addition to these correlates, we collected - in line with previous meta-analyses - moderator variables that might account for between-study differences in the effect sizes. The moderators which we used are:

a) whether emotions are conceptualized as discrete or dimensional. Whereas the former model taps into a wider spectrum of positive and negative emotions, the latter more accurately describes specific emotional reactions. We assess the magnitude of using different approaches;

b) number of items (multi-item vs. single-item scales) used to measure an emotion construct. Brown and Peterson (1993) showed that multi-item scale should yield, on average, larger effect sizes than single-item scales. Similarly, we propose that a high number of items can lead to higher effect sizes;

c) research method (observational study or experimental design). Past research showed that experimental manipulations of variables permit more control over potential confounds than traditional surveys. Experiments should produce, on average, larger effect sizes than surveys (Farley, Lehmann, and Sawyer 1995).

d) type of respondent (students vs not). Student samples have different consumption experiences and different cognitive structures (Burnett and Dunne 1986). 
e) cultural orientation. This final moderator is based on Hofstede's scores of the six dimensions of national culture. Extant literature suggests that cultural orientation shapes emotional reactions. For example:

- High individualistic cultures express emotions more intensively (Markus and Kitayama 1991).

- High power distance cultures encourage the expression of emotions that maintain status and power differences (Matsumoto 2006).

- High avoidance cultures are more emotional, (Triandis 1995).

- High masculine cultures are less emotional (Fisher et al. 2004).

- High indulgence cultures more likely remember positive emotions (Hofstede 2011).

- Long term orientation cultures are more likely to regulate emotional reactions (Matsumoto, Yoo, and Nakagawa 2008).

We control for cultural orientation, but no a priori direction is hypothesized.

\section{Method}

We conducted a keyword search on electronic databases (e.g. Proquest, EBSCO, etc.) and an Internet search using Google Scholar using as keywords the words "emotion," "affect," and "service recovery," and/or "service failure" and "complaint handling". Data collection covered the period between 2000 when the first article involving emotions in service recovery context was published, and the end of 2015. In total, we retrieved 58 usable papers providing data from 69 independent sample.

We selected the correlation coefficient as effect size metric for the meta-analysis. We converted $F$ values with one $d f$ in the numerator, and t-values into $r$ 's when necessary (Rosenthal 1991). We adjusted correlations for corrections due to measurement and sampling errors following common guidelines (Schmidt and Hunter 2014), computed the Q-test of homogeneity (Hedges and Olkin 1985), and credibility intervals (Geyskens et al. 2009) for each relationship.

We conducted a moderator analysis to evaluate the joint influence of potential moderators on the effect sizes between emotions and their correlates. We estimated a random effects metaregression model (Berkey et al. 1995) where the dependent variable $y$ is the vector of the retrieved adjusted correlation coefficients, and the independent variables are the moderators. We also included dummy variables indicating the type of construct correlated with emotions.

\section{Results}

For negative emotions, half of the studies relies on a discrete model (50.6\%), and the remaining half on a dimensional model (49.4\%). Within the discrete model, anger is the emotion that is most frequently taken into consideration (52\%), followed by frustration, regret, and helplessness. Interestingly, for positive emotions, most studies are anchored to a dimensional view model (92.7\%). Pleasure and gratitude are the only positive emotions taken into consideration as discrete constructs. Moreover, we found that discrete and dimensional constructs are measured differently. Discrete models tend to measure emotions using multi-item scales were each item represents different nuances of the same category of emotions. Dimensional models use multi-item scales where each item represents a specific type of emotion from a different emotion category.

Additionally, negative emotion scales show a wide dispersion of items expressing different types of emotions across studies, whereas this variation is less pronounced for positive emotion scales. For example, we found 25 different negative emotions mentions in the studies vs. only 13 positive ones. Anger is by far the emotion most frequently included (18.7\%) in dimensional models, followed by disappointment and annoyance (both $7.8 \%$, respectively). The most common positive emotions are happiness (20.6\%), followed by joy (12.7\%) and pleasure $(11.8 \%)$. 
We also analyzed the pairwise relationships and the average effect sizes of emotions' correlates and the results of the homogeneity analysis to test whether the observed variation in effect size values is greater than the one expected from sampling error alone. Our results show that the impact of emotions on its correlates varies substantially. First, among the three dimensions of justice, procedural justice is the dimension most strongly related to both negative $\left(r_{a d j}=-0.36\right)$ and positive emotions $\left(r_{\text {adj }}=0.48\right)$. This result highlights the central role played by the procedures used to handle the recovery in shaping emotions. Procedural justice has a stronger relationship with emotions than distributive justice. This latter was found to hold the strongest relationship with recovery satisfaction in the meta-analysis of Orsingher et al. (2011). On average, all justice dimensions have higher effect sizes for positive emotions than for negative emotions, in absolute terms. This is also true for overall justice that has the strongest relationship with both negative and positive emotions, although the magnitude is higher for positive emotions. Positive emotions have a stronger impact on satisfaction after recovery $\left(r_{a d j}=0.56\right)$ than negative emotions $\left(r_{a d j}=-0.40\right)$, although negative emotions have a strong negative impact on cumulative satisfaction $\left(\mathrm{r}_{\mathrm{adj}}=-0.58\right)$.

With respect to the relationship between emotions and outcome variables, positive emotions are more strongly related to loyalty $\left(r_{a d j}=0.48\right)$, than negative emotions $\left(r_{a d j}=-0.26\right)$. By contrast, negative emotions are more powerful in shaping WOM $\left(\mathrm{r}_{\text {adj }}=-0.30\right)$ than positive emotions $\left(\mathrm{r}_{\mathrm{adj}}=\right.$ $0.23)$. Finally, both positive and negative emotions are strongly related to trust $\left(r_{a d j}=0.49\right.$ and $r_{a d j}=$ - 0.44, respectively), although we retrieved few effect sizes for this relationship .

Regarding homogeneity analysis results are mixed. Although only five chi-square tests are significant, all credibility intervals are sufficiently wide (exceeding .11) or include zero (Sagie and Koslowsky 1993). These results suggest the presence of possible moderator variables.

Table 1 presents the results of the random effects meta-regression model estimated to evaluate the impact of possible moderators. Results show that, on average, dimensional models have higher effects sizes between emotions and their correlates. In line with our prediction, scenarios produce on average higher effects size between emotions and their correlates, whereas a large number of items generates on average lower effect sizes than a smaller number. The type of participant (students vs. non-students) does not seem to affect the size of the correlations involving emotions and the other constructs. Among the cultural values, only uncertainty avoidance and longterm orientation significantly moderate the relationships with emotion correlates.

\section{Discussion and research implications}

This meta-analysis offers the first comprehensive empirical synthesis on the role of emotions in service failure/recovery studies. First, the emotion model that scholars adopt in the different studies varies with the valence of the emotion. For negative emotions, studies are evenly distributed between the dimensional model and the discrete model of emotion. Within this latter, however, the vast majority of studies uses anger as the main negative emotion followed by frustration, regret, and helplessness. Most of the negative emotions analyzed in studies that rely on discrete models are closely related one to the other, and could be categorized - according to emotion theorists - into two basic emotions (e.g., Richins 1997). To date, we lack a comprehensive knowledge of the effect that negative emotions other than anger have on relevant outcome variables. Conversely, for positive emotions, the dimensional model of emotion predominates.

Second, we show that among the three dimensions of perceived justice, procedural justice has the strongest average correlation with both negative and positive emotions. Customers' perception of (un)fairness of recovery attributes such as the ease of engaging in the complaint process, the time required to complain and get the recovery, and the clarity of the procedures are the most powerful mechanism to make the customers happy or angry.

Third, our findings show that positive emotions are more strongly related to satisfaction after recovery and loyalty than negative emotions. These results highlight the importance of being able to provide a successful service recovery that triggers positive emotions in customers that have 
experienced a service failure. Interestingly, even if negative emotions hurt the company, their impact is less powerful than the impact of positive emotions. It appears that the service recovery process helps to mitigate the damages of negative emotions, even when it is not able to "cancel" negative feelings. This result is in line with the findings of the service recovery paradox (SRP, e.g., De Matos, Henrique and Rossi 2007), although SRP excludes customer emotions from its framework and focuses on the comparison between successful recoveries and absence of a service failure. Our results shed light on the emotional component of service failure and recovery.

Fourth, the findings of the moderator analysis indicate that the relationships between emotions and their correlates are higher when researchers use dimensional rather than discrete models of emotions. This result informs about the differences that a unidimensional versus a multidimensional view of emotional reactions produce on the relationship. More importantly, this result opens the door to the question of whether emotional reactions following service failures are better captured through a dimensional or discrete view of emotions.

Researchers should be aware that using scenarios might inflate the relationships between emotional reactions and their correlates, and that multi vs. single item scale lower on average the correlations. Researchers should be aware that using scenarios might inflate the relationships between emotional reactions and their correlates, and that multi vs. single item scale lower on average the correlations. This result, although contrary to our propositions, is not new in complaint handling studies where fewer items have been found to produce lower effects sizes (e. g. Orsingher, Valentini, and de Angelis 2010). Researchers should also acknowledge that cultural orientation produces significant differences in the magnitude of the relationship between emotions and their correlates. Uncertainty avoiding and long-term orientation cultures generate higher effect sizes.

Current study provides several managerial implications. First, we advise managers to devote particular attention to planning and communicating the recovery procedures. Attributes such as accessibility, timing, clearness of the procedures affect customer emotional states. A careful analysis and planning of the customer recovery journey might represent a fundamental first step for assuring the recovery from a bad experience.

In doing so, we suggest companies acknowledge cultural differences in the management of emotions. In particular, those companies that operate in high uncertainty avoidance cultures (e.g. Russia, Japan) and long-term orientation cultures (e.g. China, Germany) should carefully manage emotions when they design their service recovery strategies. On the one hand, customers' emotional reactions in these cultures can be difficult to identify because cultures scoring high on long-term orientation, for example, are more likely to regulate or suppress emotional reactions to preserve long-term interpersonal relationships (Matsumoto, Yoo and Nakagawa 2008). On the other hand, the impact of emotions on all the key variables associated with emotions is on average significantly higher for these cultures. Thus, identifying and managing customers' emotions in these cultures is difficult but relevant for service companies.

Second, we advise companies to provide contact employees with a comprehensive set of tools on how to manage customers' emotions. Research examining the structural dimensions of the service recovery system (e.g., Smith and Karwan 2010) has acknowledged the importance of investing resources in the human dimension. However, that research tends to emphasize more employees' ability to correct the failure, solve the problem, and discuss recovery initiatives rather than their ability to handle customers' emotional reactions. An evaluation of the effectiveness of complaint management should also include employees' ability to deal with the spectrum of emotions that arise in service failure/recovery situations.

Finally, and related to the previous point, we advise managers and supervisors to train and encourage employees that successfully manage a service recovery to display positive emotions. Research on emotional contagion (Hochshild 2003; Pugh 2001) has shown that the display of positive emotions by employees is positively related to customers' positive affect and evaluation of service quality. Thus, training employees to show happiness and pride for a successful failure resolution might be beneficial for both the customer and the service organization. 


\section{References}

Barrett, L. F. (1998). Discrete emotions or dimensions? The role of valence focus and arousal focus. Cognition \& Emotion, 12(4), 579-599.

Berkey, C. S., Hoaglin, D. C., Mosteller, F., \& Colditz, G. A. (1995). A random-effects regression model for meta-analysis. Statistics in medicine, 14(4), 395-411.

Brown, S. P., \& Peterson, R. A. (1993). Antecedents and consequences of salesperson job satisfaction: Meta-analysis and assessment of causal effects. Journal of Marketing Research, 30(1), 63-77.

Burnett, J. J., \& Dunne P.M. (1986). An Appraisal of the Use of Student Subjects in Marketing Research, Journal of Business Research, 14 (August), 329-343.

De Matos, C. A., Henrique, J. L., \& Rossi, C. A. V. (2007). Service recovery paradox: a metaanalysis. Journal of Service Research, 10(1), 60-77.

Farley, J. U., Lehmann, D. R., \& Sawyer, A. (1995). Empirical marketing generalization using meta-analysis. Marketing Science, 14(3_supplement), G36-G46.

Geyskens, I., Krishnan, R., Steenkamp, J. B. E., \& Cunha, P. V. (2009). A review and evaluation of meta-analysis practices in management research. Journal of Management, 35(2), 393-419.

Hedges, L., \& Olkin, I. (1985). Statistical models for meta-analysis. New York: Academic Press. Hedges, LV, \& Pigott, TD (2001). The power of statistical tests in meta-analysis. Psychological Methods, 6, 203-217.

Hochschild, A. R. (2003). The managed heart: Commercialization of human feeling. Univ of California Press.

Hofstede, G. (2011). Dimensionalizing cultures: The Hofstede model in context. Online readings in psychology and culture, 2(1), 8.

Schmidt, F. L., \& Hunter, J. E. (2014). Methods of meta-analysis: Correcting error and bias in research findings. Sage publications.

Laros, F. J., \& Steenkamp, J. B. E. (2005). Emotions in consumer behavior: a hierarchical approach. Journal of Business Research, 58(10), 1437-1445.

Lazarus, R. S. (1966). Psychological stress and the coping process. New York: McGraw-Hill.

Markus, H. R., \& Kitayama, S. (1991). Culture and the self: Implications for cognition, emotion, and motivation. Psychological Review, 98(2), 224-253.

Matsumoto, D. (2006). Culture and cultural worldviews: do verbal descriptions about culture reflect anything other than verbal descriptions of culture? Culture \& Psychology, 12(1), 33-62.

Matsumoto, D., Yoo, S. H., \& Nakagawa, S. (2008). Culture, emotion regulation, and adjustment. Journal of Personality and Social Psychology, 94(6), 925-937.

Orsingher, C., Valentini, S., \& De Angelis, M. (2010). A Meta-analysis of Satisfaction with Complaint Handling in Services, Journal of the Academy of Marketing Science, 38 (2), 169$186,2010$.

Plutchik, R. (1984). Emotions: A general psychoevolutionary theory. Approaches to emotion, 1984, 197-219.

Richins, M. L. (1997). Measuring emotions in the consumption experience. Journal of Consumer Research, 24(2), 127-146.

Rosenthal, R. (1991). Meta-analytic procedures for social research (Vol. 6). Sage.

Sagie, A., \& Koslowsky, M. (1993). Detecting moderators with meta-analysis: an evaluation and comparison of techniques. Personnel Psychology, 46(3), 629-640.

Smith, A. K., \& Bolton, R. N. (2002). The effect of customers' emotional responses to service failures on their recovery effort evaluations and satisfaction judgments. Journal of the Academy of Marketing Science, 30(1), 5-23.

Smith, J. S., \& Karwan, K. R. (2010). Empirical profiles of service recovery systems: the maturity perspective. Journal of Service Research, 13(1), 111-125.

Triandis, H. C. (1995). Individualism \& collectivism. Westview press. 
Table 1 Moderators Analysis: Meta-Regression Parameter Estimates

\begin{tabular}{lcc}
\hline \multicolumn{1}{c}{ Moderator } & $\begin{array}{c}\text { Unstandardized Coef. } \\
(\mathrm{SE})\end{array}$ & $p$ \\
\hline Dimensional model & $0.056(0.028)$ & 0.050 \\
Experimental design & $0.062(0.029)$ & 0.035 \\
Students & $-0.027(0.028)$ & 0.333 \\
$\mathrm{~N}$ of emotional items & $-0.039(0.006)$ & 0.000 \\
Power Distance & $0.000(0.001)$ & 0.578 \\
Individualism & $0.001(0.001)$ & 0.323 \\
Masculinity & $0.002(0.002)$ & 0.275 \\
Uncertainty Avoidance & $0.005(0.000)$ & 0.000 \\
Long Term Orientation & $0.002(0.001)$ & 0.035 \\
Indulgence & $-0.001(0.001)$ & 0.446 \\
\hline $\mathrm{N}=327, A d j . R^{2}=0.314, \mathrm{~F}_{\text {model }}[22,304]=7.80(p=0.00)$ & \\
\hline
\end{tabular}

Note: Dummy variables to control for the type of relationship were included. More specifically 12 dummy variables expressing the type of relationship (i.e. wom, return intent, loyalty, satisfaction with complaint handling, overall satisfaction, distributive, procedural, interactional, overall justices, complaint, failure, and negative emotions. 